\title{
MÉTODOS DE VARIÁVEL INSTRUMENTAL FUZZY PARA IDENTIFICAÇÃO DE SISTEMAS
}

\author{
Ginalber L.O. Serra* \\ ginalber@dmcsi.fee.unicamp.br
}

\author{
Celso P. Bottura* \\ bottura@dmcsi.fee.unicamp.br
}

* Laboratório de Controle e Sistemas Inteligentes

UNICAMP - FEEC - DMCSI

Campinas, SP, Brasil

\section{ABSTRACT}

This paper presents a theoretical proposal of fuzzy instrumental variable (FIV) methods for nonlinear discrete time systems identification and parameter estimation in noisy environment based on the fuzzy instrumental variable concept. The theoretical analysis is presented using a suitable formulation associated to Takagi-Sugeno (TS) fuzzy model. The complexity of the algorithm is quite low and the statistical properties show that the assintotic error of the parameter estimates go to zero as the data series length increases.

KEYWORDS: System identification, Fuzzy systems, Fuzzy instrumental variable.

\section{RESUMO}

Este artigo apresenta uma proposta teórica de métodos de variável instrumental fuzzy para identificação de sistemas não-lineares no tempo discreto e estimação paramétrica em ambientes ruidosos a partir do conceito de variável instrumental fuzzy. A análise teórica é apresentada a partir de uma formulação adequada associada ao modelo fuzzy

\footnotetext{
ARTIGO CONVIDADO:

Versão completa e revisada de artigo apresentado no SBAI-2005

Artigo submetido em 23/05/2006

1a. Revisão em 11/09/2006

2a. Revisão em 17/12/2006

3a. Revisão em 12/04/2007

Aceito sob recomendação do Editor Convidado

Prof. Osvaldo Ronald Saavedra Mendez
}

Takagi-Sugeno. A complexidade do algoritmo é baixa e as propriedades estatísticas mostram que o erro assintótico das estimações paramétricas tende a zero quando o conjunto de dados aumenta.

PALAVRAS-CHAVE: Identificação de sistemas, Sistemas Fuzzy, Variável instrumental fuzzy.

\section{INTRODUÇÃO}

A área de identificação de sistemas, em particular a estimação paramétrica em modelos dinâmicos, é de grande importância em muitos campos tais como engenharia de controle, análise de séries temporais, economia e processamento de sinais. Vários métodos de identificação têm sido propostos, cada um com suas vantagens e restrições, e estão em uso na comunidade científica ou até mesmo na indústria (Ljung, 1999). Atualmente existe um grande interesse em métodos paramétricos, isto é, métodos de identificação cuja parte crucial inclui a estimação paramétrica a partir de dados experimentais. As metodologias de identificação inteligente empregam técnicas motivadas por sistemas biológicos e inteligência humana e tem sido introduzidas explorando esquemas de representações alternativas usando linguagem natural, regras, redes semânticas ou modelos qualitativos (Bottura and Serra, 2004; Kasabov and Song, 2002; Serra, 2005; Serra and Bottura, 2006). Identificação fuzzy é um exemplo típico de técnicas que fazem uso de conhecimento humano e processo dedutivo. Para identificação de sistemas não-lineares, modelos fuzzy Takagi-Sugeno (TS) são 
grandemente investigados, visto que eles são aproximadores universais e fornecem boas características de interpolação e generalização(Papadakis and Theocaris, 2002; Takagi and Sugeno, 1985). Este artigo apresenta, como contribuição original, uma proposta teórica de métodos de variável instrumental fuzzy para identificação de sistemas nãolineares no tempo discreto e estimação paramétrica em ambientes ruidosos a partir do conceito de variável instrumental fuzzy proposto em ( ${ }^{a}$ Bottura and Serra, 2005; ${ }^{b}$ Bottura and Serra, 2005). A análise teórica é apresentada a partir de uma formulação adequada associada ao modelo fuzzy Takagi-Sugeno. A complexidade do algoritmo é baixa e as propriedades estatísticas mostram que o erro assintótico das estimações paramétricas tende a zero quando o conjunto de dados aumenta.

\section{MODELO FUZZY TAKAGI-SUGENO}

O sistema de inferência fuzzy Mandani, representa uma planta ou controlador por meio de regras lingüísticas SE-ENTÃO com proposições fuzzy no antecedente e no conseqüente. O sistema de inferência fuzzy TakagiSugeno (Takagi and Sugeno, 1985) usa no conseqüente uma expressão funcional das variáveis lingüísticas do antecedente. A $i$-ésima regra TS é da seguinte forma:

$$
\begin{gathered}
R^{i}: \operatorname{SE} \tilde{x}_{1} \text { é } F_{1}^{i} \text { E } \cdots \text { E } \tilde{x}_{n} \text { é } F_{n}^{i} \\
\text { ENTÃO } \tilde{y}_{i}=\left.f_{i}(\tilde{\mathbf{x}})\right|^{i=1,2, \ldots, l}
\end{gathered}
$$

onde $l$ é o número de regras. $\mathrm{O}$ vetor $\tilde{\mathbf{x}} \in$ $\Re^{n}$ contém as variáveis lingüísticas do antecedente. Cada variável lingüística tem seu próprio universo de discurso $\mathcal{U}_{\tilde{x}_{1}}, \ldots, \mathcal{U}_{\tilde{x}_{n}}$ particionados pelos conjuntos fuzzy representando os termos lingüísticos correspondentes. A variável $\tilde{x}_{j}$ pertence a um conjunto fuzzy $F_{j}^{i}$ com um valor de verdade dado por uma função de pertinência $\mu_{F_{j}}^{i}$ $: R \rightarrow[0,1]$. Esta notação refere-se ao fato de que $F_{j}^{i} \in$ $\left\{F_{j 1}^{i}, F_{j 2}^{i}, \ldots, F_{j p_{1}}^{i}\right\}$ e $\mu_{F_{j}}^{i} \in\left\{\mu_{F_{j} 1}^{i}, \mu_{F_{j} 2}^{i}, \ldots, \mu_{F_{j} p_{\tilde{x}_{j}}}^{i}\right\}$, onde $p_{\tilde{x}_{j}}$ é o número de partições do universo de discurso associado à variável lingüística $\tilde{x}_{j}$. O grau de ativação $h_{i}$ para a regra $i$ é dado por:

$$
h_{i}(\tilde{\mathbf{x}})=\mu_{F_{1}}^{i}\left(\tilde{x}_{1}^{*}\right) \star \mu_{F_{2}}^{i}\left(\tilde{x}_{2}^{*}\right) \star \ldots \star \mu_{F_{n}}^{i}\left(\tilde{x}_{n}^{*}\right)
$$

onde $\tilde{x}_{i}^{*}$ é algum ponto em $\mathcal{U}_{\tilde{x}_{i}}$ e $\star$ é uma norma-t (em nosso caso, o produto algébrico). $\mathrm{O}$ grau de ativação normalizado para a regra $i$ é definido como:

$$
\gamma_{i}(\tilde{\mathbf{x}})=\frac{h_{i}(\tilde{\mathbf{x}})}{\sum_{r=1}^{l} h_{r}(\tilde{\mathbf{x}})}
$$

Esta normalização implica em

$$
\sum_{i=1}^{l} \gamma_{i}(\tilde{\mathbf{x}})=1
$$

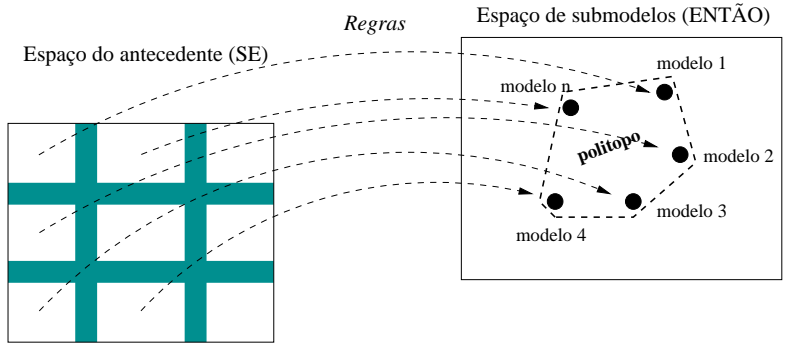

Figura 1: Mapeamento fuzzy do espaço de entrada no espaço de submodelos locais.

A resposta do modelo TS é uma soma ponderada dos conseqüentes, isto é, uma combinação convexa das funções locais (modelos) $f_{i}$ :

$$
y=\sum_{i=1}^{l} \gamma_{i}(\tilde{\mathbf{x}}) f_{i}(\tilde{\mathbf{x}})
$$

Tal modelo pode ser visto como um sistema linear variante nos parâmetros LPV (Linear Parameter Varying). Neste sentido, um modelo TS pode ser considerado como um mapeamento do espaço do antecedente (entrada) à uma região convexa (politopo) no espaço dos submodelos locais definidos pelos parâmetros do conseqüente (Bottura and Serra, 2004; Kuma, Stoll and Stoll, 2006; Skrjanc, Blazic and Agamennoni, 2005; Kukolj and Levi, 2004), conforme mostrado na Fig. 1. Esta propriedade simplifica a análise de modelos TS num contexto de sistema linear robusto tanto para identificação como para projeto de controladores com características de malha-fechada desejadas ou análise de estabilidade.

\section{FORMULAÇÃO DO PROBLEMA}

Um modelo não-linear estabelece uma relação não-linear entre a coleção de dados de entrada $u$ e saída $y$ passados e a saída predita

$$
\begin{gathered}
\hat{y}(k+1)=\tilde{F}\left(y(k), \ldots, y\left(k-n_{y}+1\right), u(k), \ldots\right. \\
\left.\ldots, u\left(k-n_{u}+1\right)\right)
\end{gathered}
$$

onde $k$ é o instante de amostragem, $n_{y}$ e $n_{u}$ são inteiros relacionados à ordem do sistema. Neste traballho, será estabelecida a estrutura de modelo autorregressivo com entrada exógena não-linear NARX (Nonlinear AutoRegressive with eXogenous input), usada na maioria dos métodos de identificação não-linear tais como redes neurais, funções de base radial, CMAC (Cerebellar Model Articulation Controller), e também modelos fuzzy (Brown and Harris, 1994; Tsoukalas and Uhrig, 1997). Com uma 
formulação baseada no modelo fuzzy Takagi-Sugeno, tem-se

$$
\begin{gathered}
R^{i}: \mathrm{SE} y(k) \text { é } F_{1}^{i} \mathrm{E} \cdots \mathrm{E} y\left(k-n_{y}+1\right) \text { é } \\
F_{n_{y}}^{i} \mathrm{E} u(k) \text { é } G_{1}^{i} \mathrm{E} \cdots \mathrm{E} u\left(k-n_{u}+1\right) \\
\text { é } G_{n_{u}}^{i} \operatorname{ENTÃO} \hat{y}_{i}(k+1)=\sum_{j=1}^{n_{y}} a_{i, j} y(k-j+1) \\
+\sum_{j=1}^{n_{u}} b_{i, j} u(k-j+1)+c_{i}
\end{gathered}
$$

onde $a_{i, j}, b_{i, j}$ e $c_{i}$ são os parâmetros do conseqüente a serem determinados. A fórmula de inferência é dada por

$$
\hat{y}(k+1)=\frac{\sum_{i=1}^{l} h_{i}\left(\mathbf{x}_{k}\right) \hat{y}_{i}(k+1)}{\sum_{i=1}^{l} h_{i}\left(\mathbf{x}_{k}\right)}
$$

$\mathrm{ou}$

$$
\hat{y}(k+1)=\sum_{i=1}^{l} \gamma_{i}\left(\mathbf{x}_{k}\right) \hat{y}_{i}(k+1)
$$

com

$$
\begin{aligned}
\mathbf{x}_{k}= & \left(y(k), \ldots, y\left(k-n_{y}+1\right), u(k), \ldots\right. \\
& \left.\ldots, u\left(k-n_{u}+1\right)\right)
\end{aligned}
$$

Este modelo NARX representa sistemas de múltiplas entradas e única saída MISO (Multiple Input Single Output) diretamente e sistemas de múltiplas entradas e múltiplas saídas MIMO (Multiple Input Multiple Output) em uma forma decomposta como um conjunto de modelos MISO acoplados.

\subsection{Problema de estimação parâmetros do conseqüente}

A fórmula de inferência do modelo fuzzy em (9) pode ser expressa como

$$
\begin{aligned}
& \hat{y}(k+1)=\gamma_{1}\left(\mathbf{x}_{k}\right)\left[a_{1,1} y(k)+\ldots+a_{1, n y} y\left(k-n_{y}\right.\right. \\
& \left.+1)+b_{1,1} u(k)+\ldots+b_{1, n u} u\left(k-n_{u}+1\right)+c_{1}\right]+ \\
& +\gamma_{2}\left(\mathbf{x}_{k}\right)\left[a_{2,1} y(k)+\ldots+a_{2, n y} y\left(k-n_{y}+1\right)+\right. \\
& \left.+b_{2,1} u(k)+\ldots+b_{2, \text { пu }} u\left(k-n_{u}+1\right)+c_{2}\right]+\ldots
\end{aligned}
$$

dos

$$
\begin{gathered}
+\gamma_{l}\left(\mathbf{x}_{k}\right)\left[a_{l, 1} y(k)+\ldots+a_{l, n y} y\left(k-n_{y}+1\right)+\right. \\
\left.+b_{l, 1} u(k)+\ldots+b_{l, n u} u\left(k-n_{u}+1\right)+c_{l}\right]
\end{gathered}
$$

que é linear nos parâmetros do conseqüente, $\mathbf{a}, \mathbf{b}$ e $\mathbf{c}$. Para um conjunto de $N$ pares de dados de entrada-saída $\left\{\left(\mathbf{u}_{k}, y_{k}\right) \mid i=1,2, \ldots, N\right\}$ disponível, a seguinte forma vetorial é obtida

$$
\mathbf{Y}=\left[\psi_{1} \mathbf{X}, \psi_{2} \mathbf{X}, \ldots, \psi_{l} \mathbf{X}\right] \boldsymbol{\Theta}+\boldsymbol{\Xi}
$$

onde $\psi_{i}=\operatorname{diag}\left(\gamma_{i}\left(\mathbf{x}_{k}\right)\right) \in \Re^{N \times N}, \mathbf{X}=\left[\mathbf{y}_{k}, \ldots\right.$, $\left.\mathbf{y}_{k-n y+1}, \mathbf{u}_{k}, \ldots, \mathbf{u}_{k-n u+1}, \mathbf{1}\right] \in \Re^{N \times\left(n_{y}+n_{u}+1\right)}, \mathbf{Y} \in$ $\Re^{N \times 1}, \boldsymbol{\Xi} \in \Re^{N \times 1}$ e $\boldsymbol{\Theta} \in \Re^{l\left(n_{y}+n_{u}+1\right) \times 1}$ são a matriz de grau de pertinência normalizada, a matriz de dados, o vetor de saída, o vetor de ruído desconhecido e o vetor de parâmetros estimados, respectivamente.

Diferentes técnicas podem ser usadas quando as variáveis associadas com os parâmetros desconhecidos são exatamente conhecidas. Na prática, e no presente contexto, as relações básicas entre os parâmetros ainda estão na forma apresentada em (12), mas os elementos de $\mathbf{X}$ não são exatamente conhecidos e podem ser observados com erro de maneira que o valor observado é expresso por

$$
y_{k}=\chi_{k}^{T} \theta+\eta_{k}
$$

onde, no $k$-ésimo instante de amostragem, $\chi_{k}^{T}=\left[\gamma_{k}^{1}\left(\mathbf{x}_{k}+\right.\right.$ $\left.\left.\xi_{k}\right), \ldots, \gamma_{k}^{l}\left(\mathbf{x}_{k}+\xi_{k}\right)\right]$ é o vetor de dados com erro nas variáveis, $\mathbf{x}_{k}=\left[y_{k-1}, \ldots, y_{k-n_{y}}, u_{k-1}, \ldots, u_{k-n_{u}}, 1\right]^{T}$ é o vetor de dados com quantidades exatamente conhecidas, isto é, dados de entrada-saída livres de ruído, $\xi_{k}$ é um vetor de ruído associado com a observação de $\mathbf{x}_{k}$, e $\eta_{k}$ é um ruído de saída.

As equações normais são formuladas por

$$
\left[\sum_{j=1}^{k} \chi_{j} \chi_{j}^{T}\right] \hat{\theta}_{k}=\sum_{j=1}^{k} \chi_{j} y_{j}
$$

e multiplicando por $\frac{1}{k}$ resulta

$$
\begin{gathered}
\left\{\frac { 1 } { k } \sum _ { j = 1 } ^ { k } [ \gamma _ { j } ^ { 1 } ( \mathbf { x } _ { j } + \xi _ { j } ) , \ldots , \gamma _ { j } ^ { l } ( \mathbf { x } _ { j } + \xi _ { j } ) ] \left[\gamma_{j}^{1}\left(\mathbf{x}_{j}+\xi_{j}\right),\right.\right. \\
\left.\left.\ldots, \gamma_{j}^{l}\left(\mathbf{x}_{j}+\xi_{j}\right)\right]^{T}\right\} \hat{\theta}_{k}=\frac{1}{k} \sum_{j=1}^{k}\left[\gamma_{j}^{1}\left(\mathbf{x}_{j}+\xi_{j}\right), \ldots,\right. \\
\left.\gamma_{j}^{l}\left(\mathbf{x}_{j}+\xi_{j}\right)\right] y_{j}
\end{gathered}
$$

Visto que $y_{j}=\chi_{j}^{T} \theta+\eta_{j}$,

$$
\left\{\frac { 1 } { k } \sum _ { j = 1 } ^ { j } [ \gamma _ { j } ^ { 1 } ( \mathbf { x } _ { j } + \xi _ { j } ) , \ldots , \gamma _ { j } ^ { l } ( \mathbf { x } _ { j } + \xi _ { j } ) ] \left[\gamma_{j}^{1}\left(\mathbf{x}_{j}+\xi_{j}\right),\right.\right.
$$




$$
\begin{gathered}
\left.\left.\ldots, \gamma_{j}^{l}\left(\mathbf{x}_{j}+\xi_{j}\right)\right]^{T}\right\} \hat{\theta}_{k}=\frac{1}{k} \sum_{j=1}^{k}\left[\gamma_{j}^{1}\left(\mathbf{x}_{j}+\xi_{j}\right), \ldots,\right. \\
\left.\gamma_{j}^{l}\left(\mathbf{x}_{j}+\xi_{j}\right)\right]\left[\gamma_{j}^{1}\left(\mathbf{x}_{j}+\xi_{j}\right), \ldots, \gamma_{j}^{l}\left(\mathbf{x}_{j}+\xi_{j}\right)\right]^{T} \theta+ \\
\quad+\frac{1}{k} \sum_{j=1}^{k}\left[\gamma_{j}^{1}\left(\mathbf{x}_{j}+\xi_{j}\right), \ldots, \gamma_{j}^{l}\left(\mathbf{x}_{j}+\xi_{j}\right)\right] \eta_{j}
\end{gathered}
$$

e

$$
\begin{gathered}
\tilde{\theta}_{k}=\left[\frac { 1 } { k } \sum _ { j = 1 } ^ { k } [ \gamma _ { j } ^ { 1 } ( \mathbf { x } _ { j } + \xi _ { j } ) , \ldots , \gamma _ { j } ^ { l } ( \mathbf { x } _ { j } + \xi _ { j } ) ] \left[\gamma _ { j } ^ { 1 } \left(\mathbf{x}_{j}+\right.\right.\right. \\
\left.\left.\left.+\xi_{j}\right), \ldots, \gamma_{j}^{l}\left(\mathbf{x}_{j}+\xi_{j}\right)\right]^{T}\right]^{-1} \frac{1}{k} \sum_{j=1}^{k}\left[\gamma_{j}^{1}\left(\mathbf{x}_{j}+\xi_{j}\right), \ldots\right. \\
\left.\ldots, \gamma_{j}^{l}\left(\mathbf{x}_{j}+\xi_{j}\right)\right] \eta_{j}
\end{gathered}
$$

onde $\tilde{\theta}_{k}=\hat{\theta}_{k}-\theta$ é o erro paramétrico. Tomando-se a probabilidade no limite, quando $k \rightarrow \infty$,

$$
\text { p. } \lim _{k \rightarrow \infty} \tilde{\theta}_{k}=\text { p. } \lim _{k \rightarrow \infty}\left\{\frac{1}{k} \mathbf{C}_{k}^{-1} \frac{1}{k} \mathbf{b}_{k}\right\}
$$

com

$$
\begin{gathered}
\mathbf{C}_{k}=\sum_{j=1}^{k}\left[\gamma_{j}^{1}\left(\mathbf{x}_{j}+\xi_{j}\right), \ldots, \gamma_{j}^{l}\left(\mathbf{x}_{j}+\xi_{j}\right)\right]\left[\gamma _ { j } ^ { 1 } \left(\mathbf{x}_{j}+\right.\right. \\
\left.\left.+\xi_{j}\right), \ldots, \gamma_{j}^{l}\left(\mathbf{x}_{j}+\xi_{j}\right)\right]^{T} \\
\mathbf{b}_{k}=\sum_{j=1}^{k}\left[\gamma_{j}^{1}\left(\mathbf{x}_{j}+\xi_{j}\right), \ldots, \gamma_{j}^{l}\left(\mathbf{x}_{j}+\xi_{j}\right)\right] \eta_{j}
\end{gathered}
$$

Aplicando-se o teorema de Slutsky (Goodwin and Payne, 1977) e supondo que os elementos de $\frac{1}{k} \mathbf{C}_{k}$ e $\frac{1}{k} \mathbf{b}_{k}$ convergem em probabilidade, temos

$$
\text { p. } \lim _{k \rightarrow \infty} \tilde{\theta}_{k}=\text { p. } \lim _{k \rightarrow \infty} \frac{1}{k} \mathbf{C}_{k}^{-1} \text { p. } \lim _{k \rightarrow \infty} \frac{1}{k} \mathbf{b}_{k}
$$

Assim,

$$
\begin{aligned}
& \text { p. } \lim _{k \rightarrow \infty} \frac{1}{k} \mathbf{C}_{k}=\text { p. } \lim _{k \rightarrow \infty} \frac{1}{k} \sum_{j=1}^{k}\left[\gamma_{j}^{1}\left(\mathbf{x}_{j}+\xi_{j}\right), \ldots, \gamma_{j}^{l}\left(\mathbf{x}_{j}+\right.\right. \\
& \left.\left.+\xi_{j}\right)\right]\left[\gamma_{j}^{1}\left(\mathbf{x}_{j}+\xi_{j}\right), \ldots, \gamma_{j}^{l}\left(\mathbf{x}_{j}+\xi_{j}\right)\right]^{T} \\
& \text { p. } \lim _{k \rightarrow \infty} \frac{1}{k} \mathbf{C}_{k}=\text { p. } \lim _{k \rightarrow \infty} \frac{1}{k} \sum_{j=1}^{k}\left(\gamma_{j}^{1}\right)^{2}\left(\mathbf{x}_{j}+\xi_{j}\right)\left(\mathbf{x}_{j}+\xi_{j}\right)^{T} \\
& +\ldots+\text { p. } \lim _{k \rightarrow \infty} \frac{1}{k} \sum_{j=1}^{k}\left(\gamma_{j}^{l}\right)^{2}\left(\mathbf{x}_{j}+\xi_{j}\right)\left(\mathbf{x}_{j}+\xi_{j}\right)^{T}
\end{aligned}
$$

Supondo $\mathbf{x}_{j}$ e $\xi_{j}$ estatisticamente independentes,

$$
\begin{gathered}
\text { p. } \lim _{k \rightarrow \infty} \frac{1}{k} \mathbf{C}_{k}=\text { p. } \lim _{k \rightarrow \infty} \frac{1}{k} \sum_{j=1}^{k}\left(\gamma_{j}^{1}\right)^{2}\left[\mathbf{x}_{j} \mathbf{x}_{j}^{T}+\xi_{j} \xi_{j}^{T}\right]+\ldots+ \\
+ \text { p. } \lim _{k \rightarrow \infty} \frac{1}{k} \sum_{j=1}^{k}\left(\gamma_{j}^{l}\right)^{2}\left[\mathbf{x}_{j} \mathbf{x}_{j}^{T}+\xi_{j} \xi_{j}^{T}\right] \\
\text { p. } \lim _{k \rightarrow \infty} \frac{1}{k} \mathbf{C}_{k}=\text { p. } \lim _{k \rightarrow \infty} \frac{1}{k} \sum_{j=1}^{k} \mathbf{x}_{j} \mathbf{x}_{j}^{T}\left[\left(\gamma_{j}^{1}\right)^{2}+\ldots+\left(\gamma_{j}^{l}\right)^{2}\right] \\
+ \text { p. } \lim _{k \rightarrow \infty} \frac{1}{k} \sum_{j=1}^{k} \xi_{j} \xi_{j}^{T}\left[\left(\gamma_{j}^{1}\right)^{2}+\ldots+\left(\gamma_{j}^{l}\right)^{2}\right]
\end{gathered}
$$

$\operatorname{com} \sum_{i=1}^{l} \gamma_{j}^{i}=1$. Logo, a análise assintótica da estimação dos parâmetros do consequiente associada a modelos fuzzy TS é baseada na soma ponderada das matrizes de covariância de $\mathrm{x}$ e $\xi$. Similarmente,

$$
\begin{aligned}
& \text { p. } \lim _{k \rightarrow \infty} \frac{1}{k} \mathbf{b}_{k}=\text { p. } \lim _{k \rightarrow \infty} \frac{1}{k} \sum_{j=1}^{k}\left[\gamma_{j}^{1}\left(\mathbf{x}_{j}+\xi_{j}\right), \ldots, \gamma_{j}^{l}\left(\mathbf{x}_{j}+\right.\right. \\
& \left.\left.+\xi_{j}\right)\right] \eta_{j} \\
& \text { p. } \lim _{k \rightarrow \infty} \frac{1}{k} \mathbf{b}_{k}=\text { p. } \lim _{k \rightarrow \infty} \frac{1}{k} \sum_{j=1}^{k}\left[\gamma_{j}^{1} \xi_{j} \eta_{j}, \ldots, \gamma_{j}^{l} \xi_{j} \eta_{j}\right]
\end{aligned}
$$

Substituindo-se (20) e (21) em (19), resulta

$$
\begin{gathered}
\text { p. } \lim _{k \rightarrow \infty} \tilde{\theta}_{k}=\left\{\text { p. } \lim _{k \rightarrow \infty} \frac{1}{k} \sum_{j=1}^{k} \mathbf{x}_{j} \mathbf{x}_{j}^{T}\left[\left(\gamma_{j}^{1}\right)^{2}+\ldots+\left(\gamma_{j}^{l}\right)^{2}\right]+\right. \\
\left.+ \text { p. } \lim _{k \rightarrow \infty} \frac{1}{k} \sum_{j=1}^{k} \xi_{j} \xi_{j}^{T}\left[\left(\gamma_{j}^{1}\right)^{2}+\ldots+\left(\gamma_{j}^{l}\right)^{2}\right]\right\}^{-1} \text { p. } \lim _{k \rightarrow \infty} \frac{1}{k} \\
\sum_{j=1}^{k}\left[\gamma_{j}^{1} \xi_{j} \eta_{j}, \ldots, \gamma_{j}^{l} \xi_{j} \eta_{j}\right]
\end{gathered}
$$

com $\sum_{i=1}^{l} \gamma_{j}^{i}=1$. Para o caso de apenas uma regra $(l=1)$, a análise é simplificada ao modelo linear, com $\left.\gamma_{j}^{i}\right|_{j=1, \ldots, k} ^{i=1}=1$. Visto que a entrada $u_{k}$ continua a excitar o processo e, ao mesmo tempo, os coeficientes nos submodelos do conseqüente não são todos nulos, então a saída $y_{k}$ existirá para todo intervalo de observação $k$. Conseqüentemente, a matriz de covariância $\mathbf{x}_{j} \mathbf{x}_{j}^{T}$ também será não-singular e a sua inversa existirá. Assim, o único modo no qual o erro paramétrico será nulo é para $\xi_{j} \eta_{j}$ zero. Mas, em geral, $\xi_{j}$ e $\eta_{j}$ estão correlacionados e o erro assintótico não será zero e a estimação de mínimos quadrados será polarizada definida pela taxa relativa da variância sinal/ruído. Em outras palavras, a estimação de mínimos quadrados não é apropriada para estimar os parâmetros do conseqüente pois a estimação será inconsistente e a polarização permanecerá independentemente do número de dados usados na estimação. 


\section{VARIÁVEL INSTRUMENTAL FUZZY}

Em identificação de sistemas usando modelos lineares, para baixos níveis de ruído, o método de mínimos quadrados apresenta desempenho satisfatório na estimação dos parâmetros do modelo. Na presença de ruído colorido, o método dos mínimos quadrados fornece uma estimativa polarizada, ou seja, ao se fazer muitas observações a estimativa não se aproxima do valor verdadeiro dos parâmetros. Para níveis maiores de ruído, ou na presença de ruído colorido, métodos tais como GLS (Generalized Least Squares) ( ${ }^{a}$ Söderström and Stoica, 1989), ELS (Extended Least Squares) (Ljung and Söderström, 1987) e PE (Prediction Error) (Ljung, 1999; Young, 1984) podem ser usados, embora sejam inevitavelmente dependentes da precisão do modelo do ruído. Para superar o problema de polarização e inconsistência da estimação paramétrica, sem a necessidade de modelar o ruído, o método IV (Instrumental Variable) pode ser usado $\left({ }^{b}\right.$ Söderström and Stoica, 1983). Neste método, um vetor estatisticamente independente dos ruídos e correlacionado com o vetor de dados do sistema $\mathbf{x}_{j}$ se faz necessário. Se isto é possível, então será fácil ver que a escolha deste vetor é eficiente para remover a polarização assintótica da estimação dos parâmetros do conseqüente. A estimação fuzzy no sentido dos mínimos quadrados é dada por:

$$
\begin{gathered}
\left\{\frac { 1 } { k } \sum _ { j = 1 } ^ { k } [ \gamma _ { j } ^ { 1 } ( \mathbf { x } _ { j } + \xi _ { j } ) , \ldots , \gamma _ { j } ^ { l } ( \mathbf { x } _ { j } + \xi _ { j } ) ] \left[\gamma_{j}^{1}\left(\mathbf{x}_{j}+\xi_{j}\right),\right.\right. \\
\left.\left.\ldots, \gamma_{j}^{l}\left(\mathbf{x}_{j}+\xi_{j}\right)\right]^{T}\right\} \hat{\theta}_{k}=\frac{1}{k} \sum_{j=1}^{k}\left[\gamma_{j}^{1}\left(\mathbf{x}_{j}+\xi_{j}\right), \ldots,\right. \\
\left.\gamma_{j}^{l}\left(\mathbf{x}_{j}+\xi_{j}\right)\right]\left\{\left[\gamma_{j}^{1}\left(\mathbf{x}_{j}+\xi_{j}\right), \ldots, \gamma_{j}^{l}\left(\mathbf{x}_{j}+\xi_{j}\right)\right]^{T} \theta+\eta_{j}\right\}
\end{gathered}
$$

Usando-se um novo vetor de variáveis lingüísticas $\left[\beta_{j}^{1} \mathbf{z}_{j}, \ldots, \beta_{j}^{l} \mathbf{z}_{j}\right]$, a última equação fica da forma

$$
\begin{gathered}
\left\{\frac { 1 } { k } \sum _ { j = 1 } ^ { k } [ \beta _ { j } ^ { 1 } \mathbf { z } _ { j } , \ldots , \beta _ { j } ^ { l } \mathbf { z } _ { j } ] \left[\gamma_{j}^{1}\left(\mathbf{x}_{j}+\xi_{j}\right), \ldots, \gamma_{j}^{l}\left(\mathbf{x}_{j}+\right.\right.\right. \\
\left.\left.\left.+\xi_{j}\right)\right]^{T}\right\} \hat{\theta}_{k}=\frac{1}{k} \sum_{j=1}^{k}\left[\beta_{j}^{1} \mathbf{z}_{j}, \ldots, \beta_{j}^{l} \mathbf{z}_{j}\right]\left\{\left[\gamma_{j}^{1}\left(\mathbf{x}_{j}+\xi_{j}\right),\right.\right. \\
\left.\left.\ldots, \gamma_{j}^{l}\left(\mathbf{x}_{j}+\xi_{j}\right)\right]^{T} \theta+\eta_{j}\right\}
\end{gathered}
$$

onde $\mathbf{z}_{j}$ é um vetor com a ordem de $\mathbf{x}_{j}$, associado ao comportamento dinâmico do sistema, e $\left.\beta_{j}^{i}\right|^{i=1, \ldots, l}$ é o grau de ativação normalizado, como em (3), associado a $\mathbf{z}_{j}$. Para análise de convergência, são propostos os seguintes lemas e teorema:

Lema 1 Seja $\mathbf{z}_{j}$ um vetor com a ordem de $\mathbf{x}_{j}$, associado ao comportamento dinâmico do sistema e independente da entrada de ruído $\xi_{j}$, e $\left.\beta_{j}^{i}\right|^{i=1, \ldots, l}$ é o grau de ativação normalizado, como em (3), associado a $\mathbf{z}_{j}$. Então, no limite

$$
\lim _{k \rightarrow \infty} \frac{1}{k} \sum_{j=1}^{k}\left[\beta_{j}^{1} \mathbf{z}_{j}, \ldots, \beta_{j}^{l} \mathbf{z}_{j}\right] \xi_{j}^{T}=\mathbf{0}
$$

Prova: Desenvolvendo-se o lado esquerdo de (24), resulta

$$
\begin{gathered}
\lim _{k \rightarrow \infty} \frac{1}{k} \sum_{j=1}^{k}\left[\beta_{j}^{1} \mathbf{z}_{j}, \ldots, \beta_{j}^{l} \mathbf{z}_{j}\right] \xi_{j}^{T}=\lim _{k \rightarrow \infty} \frac{1}{k} \sum_{j=1}^{k}\left[\beta_{j}^{1} \mathbf{z}_{j} \xi_{j}^{T}\right. \\
\left.\ldots, \beta_{j}^{l} \mathbf{z}_{j} \xi_{j}^{T}\right]
\end{gathered}
$$

Como $\left.\beta_{j}^{i}\right|^{i=1, \ldots, l}$ é um escalar, e, por definição, o vetor de variáveis escolhido é estatisticamente independente das entradas ruidosas, o produto interno entre $\mathbf{z}_{j}$ e $\xi_{j}$ será zero. Assim, tomando-se o limite, resulta

$$
\lim _{k \rightarrow \infty} \frac{1}{k} \sum_{j=1}^{k}\left[\beta_{j}^{1} \mathbf{z}_{j} \xi_{j}^{T}, \ldots, \beta_{j}^{l} \mathbf{z}_{j} \xi_{j}^{T}\right]=\mathbf{0}
$$

Lema 2 Seja $\mathbf{z}_{j}$ um vetor com a ordem de $\mathbf{x}_{j}$, associado ao comportamento dinâmico do sistema e independente do ruído $\eta_{j}$, e $\left.\beta_{j}^{i}\right|^{i=1, \ldots, l}$ o grau de ativação normalizado, como em (3), associado a $\mathbf{z}_{j}$. Então, no limite

$$
\lim _{k \rightarrow \infty} \frac{1}{k} \sum_{j=1}^{k}\left[\beta_{j}^{1} \mathbf{z}_{j}, \ldots, \beta_{j}^{l} \mathbf{z}_{j}\right] \eta_{j}=\mathbf{0}
$$

Prova: Desenvolvendo o lado esquerdo de (25), resulta

$$
\begin{gathered}
\lim _{k \rightarrow \infty} \frac{1}{k} \sum_{j=1}^{k}\left[\beta_{j}^{1} \mathbf{z}_{j}, \ldots, \beta_{j}^{l} \mathbf{z}_{j}\right] \eta_{j}=\lim _{k \rightarrow \infty} \frac{1}{k} \sum_{j=1}^{k}\left[\beta_{j}^{1} \mathbf{z}_{j} \eta_{j}\right. \\
\left.\ldots, \beta_{j}^{l} \mathbf{z}_{j} \eta_{j}\right]
\end{gathered}
$$

Visto que o vetor de variáveis escolhido é estatisticamente independente do ruído de observação, o produto entre $\mathbf{z}_{j}$ e $\eta_{j}$ será zero no limite. Logo,

$$
\lim _{k \rightarrow \infty} \frac{1}{k} \sum_{j=1}^{k}\left[\beta_{j}^{1} \mathbf{z}_{j} \eta_{j}, \ldots, \beta_{j}^{l} \mathbf{z}_{j} \eta_{j}\right]=\mathbf{0}
$$

Lema 3 Seja $\mathbf{z}_{j}$ um vetor com a ordem de $\mathbf{x}_{j}$, associado ao comportamento dinâmico do sistema, e $\left.\beta_{j}^{i}\right|^{i=1, \ldots, l}$ é o grau de ativação normalizado, como em (3), associado a $\mathbf{z}_{j}$. Então, de acordo com (23), no limite

$$
\lim _{k \rightarrow \infty} \frac{1}{k} \sum_{j=1}^{k}\left[\beta_{j}^{1} \mathbf{z}_{j}, \ldots, \beta_{j}^{l} \mathbf{z}_{j}\right]\left[\gamma_{j}^{1}\left(\mathbf{x}_{j}+\xi_{j}\right), \ldots, \gamma_{j}^{l}\left(\mathbf{x}_{j}\right.\right.
$$




$$
\left.\left.+\xi_{j}\right)\right]^{T}=\mathbf{C}_{\mathbf{z x}} \neq 0
$$

Prova: Desenvolvendo o lado esquerdo de (26), resulta

$$
\begin{gathered}
\lim _{k \rightarrow \infty} \frac{1}{k} \sum_{j=1}^{k}\left[\beta_{j}^{1} \mathbf{z}_{j}, \ldots, \beta_{j}^{l} \mathbf{z}_{j}\right]\left[\gamma_{j}^{1}\left(\mathbf{x}_{j}+\xi_{j}\right), \ldots, \gamma_{j}^{l}\left(\mathbf{x}_{j}\right.\right. \\
\left.\left.+\xi_{j}\right)\right]^{T}=\lim _{k \rightarrow \infty} \frac{1}{k} \sum_{j=1}^{k}\left[\beta_{j}^{1} \gamma_{j}^{1}\left(\mathbf{z}_{j} \mathbf{x}_{j}^{T}+\mathbf{z}_{j} \xi_{j}^{T}\right)+\ldots+\right. \\
\left.+\beta_{j}^{l} \gamma_{j}^{l}\left(\mathbf{z}_{j} \mathbf{x}_{j}^{T}+\mathbf{z}_{j} \xi_{j}^{T}\right)\right]
\end{gathered}
$$

A partir do Lema 1, esta expressão é simplificada para

$$
\begin{aligned}
& \lim _{k \rightarrow \infty} \frac{1}{k} \sum_{j=1}^{k}\left[\beta_{j}^{1} \mathbf{z}_{j}, \ldots, \beta_{j}^{l} \mathbf{z}_{j}\right]\left[\gamma_{j}^{1}\left(\mathbf{x}_{j}+\xi_{j}\right), \ldots, \gamma_{j}^{l}\left(\mathbf{x}_{j}\right.\right. \\
& \left.\left.+\xi_{j}\right)\right]^{T}=\lim _{k \rightarrow \infty} \frac{1}{k} \sum_{j=1}^{k}\left[\beta_{j}^{1} \gamma_{j}^{1} \mathbf{z}_{j} \mathbf{x}_{j}^{T}+\ldots+\beta_{j}^{l} \gamma_{j}^{l} \mathbf{z}_{j} \mathbf{x}_{j}^{T}\right]
\end{aligned}
$$

e esta matriz de covariância tem a seguinte propriedade:

$$
\lim _{k \rightarrow \infty} \frac{1}{k} \sum_{j=1}^{k}\left[\beta_{j}^{1} \gamma_{j}^{1} \mathbf{z}_{j} \mathbf{x}_{j}^{T}+\ldots+\beta_{j}^{l} \gamma_{j}^{l} \mathbf{z}_{j} \mathbf{x}_{j}^{T}\right] \neq 0
$$

e

$$
\lim _{k \rightarrow \infty} \frac{1}{k} \sum_{j=1}^{k}\left[\beta_{j}^{1} \gamma_{j}^{1} \mathbf{z}_{j} \mathbf{x}_{j}^{T}+\ldots+\beta_{j}^{l} \gamma_{j}^{l} \mathbf{z}_{j} \mathbf{x}_{j}^{T}\right]=\mathbf{C}_{\mathbf{z x}} \neq \mathbf{0}
$$

Teorema 1 Seja $\mathbf{z}_{j}$ um vetor com a ordem de $\mathbf{x}_{j}$, associado ao comportamento dinâmico do sistema, e $\left.\beta_{j}^{i}\right|^{i=1, \ldots, l}$ é o grau de ativação normalizado, como em (3), associado a $\mathbf{z}_{j}$. Então, no limite

$$
p . \lim _{k \rightarrow \infty} \tilde{\theta}=0
$$

Prova: A partir do novo vetor de variáveis lingüísticas $\left[\beta_{j}^{1} \mathbf{z}_{j}, \ldots, \beta_{j}^{l} \mathbf{z}_{j}\right], \quad$ a estimação dos parâmetros do consequiente, no sentido dos mínimos quadrados, pode ser modificada como segue:

$$
\begin{gathered}
\left\{\frac { 1 } { k } \sum _ { j = 1 } ^ { k } [ \beta _ { j } ^ { 1 } \mathbf { z } _ { j } , \ldots , \beta _ { j } ^ { l } \mathbf { z } _ { j } ] \left[\gamma_{j}^{1}\left(\mathbf{x}_{j}+\xi_{j}\right), \ldots, \gamma_{j}^{l}\left(\mathbf{x}_{j}+\right.\right.\right. \\
\left.\left.\left.+\xi_{j}\right)\right]^{T}\right\} \hat{\theta}_{k}=\frac{1}{k} \sum_{j=1}^{k}\left[\beta_{j}^{1} \mathbf{z}_{j}, \ldots, \beta_{j}^{l} \mathbf{z}_{j}\right]\left\{\left[\gamma_{j}^{1}\left(\mathbf{x}_{j}+\xi_{j}\right),\right.\right. \\
\left.\left.\ldots, \gamma_{j}^{l}\left(\mathbf{x}_{j}+\xi_{j}\right)\right]^{T} \theta+\eta_{j}\right\}
\end{gathered}
$$

a qual pode ser expressa como

$$
\left\{\frac { 1 } { k } \sum _ { j = 1 } ^ { k } [ \beta _ { j } ^ { 1 } \mathbf { z } _ { j } , \ldots , \beta _ { j } ^ { l } \mathbf { z } _ { j } ] \left[\gamma_{j}^{1}\left(\mathbf{x}_{j}+\xi_{j}\right), \ldots, \gamma_{j}^{l}\left(\mathbf{x}_{j}+\right.\right.\right.
$$

$$
\left.\left.\left.+\xi_{j}\right)\right]^{T}\right\}\left(\hat{\theta}_{k}-\theta\right)=\frac{1}{k} \sum_{j=1}^{k}\left[\beta_{j}^{1} \mathbf{z}_{j}, \ldots, \beta_{j}^{l} \mathbf{z}_{j}\right] \eta_{j}
$$

e

$$
\begin{gathered}
\left\{\frac { 1 } { k } \sum _ { j = 1 } ^ { k } [ \beta _ { j } ^ { 1 } \mathbf { z } _ { j } , \ldots , \beta _ { j } ^ { l } \mathbf { z } _ { j } ] \left[\gamma_{j}^{1}\left(\mathbf{x}_{j}+\xi_{j}\right), \ldots, \gamma_{j}^{l}\left(\mathbf{x}_{j}+\right.\right.\right. \\
\left.\left.\left.+\xi_{j}\right)\right]^{T}\right\} \tilde{\theta}=\frac{1}{k} \sum_{j=1}^{k}\left[\beta_{j}^{1} \mathbf{z}_{j}, \ldots, \beta_{j}^{l} \mathbf{z}_{j}\right] \eta_{j}
\end{gathered}
$$

Tomando-se a probabilidade no limite com $k \rightarrow \infty$, e aplicando-se o teorema de Slutsky, temos

$$
\begin{gathered}
\text { p. } \lim _{k \rightarrow \infty} \tilde{\theta}_{k}=\left\{\text { p. } \operatorname { l i m } _ { k \rightarrow \infty } \frac { 1 } { k } \sum _ { j = 1 } ^ { k } [ \beta _ { j } ^ { 1 } \mathbf { z } _ { j } , \ldots , \beta _ { j } ^ { l } \mathbf { z } _ { j } ] \left[\gamma_{j}^{1}\left(\mathbf{x}_{j}+\xi_{j}\right), \ldots,\right.\right. \\
\left.\left.\gamma_{j}^{l}\left(\mathbf{x}_{j}+\xi_{j}\right)\right]^{T}\right\}^{-1}\left\{\text { p. } \lim _{k \rightarrow \infty} \frac{1}{k} \sum_{j=1}^{k}\left[\beta_{j}^{1} \mathbf{z}_{j}, \ldots, \beta_{j}^{l} \mathbf{z}_{j}\right] \eta_{j}\right\}
\end{gathered}
$$

De acordo com o Lema 1 e o Lema 3, resulta

$$
\begin{gathered}
\text { p. } \lim _{k \rightarrow \infty} \tilde{\theta}_{k}=\left\{\mathrm{p} . \lim _{k \rightarrow \infty} \mathbf{C}_{\mathbf{z x}}\right\}^{-1}\left\{\mathrm{p} . \lim _{k \rightarrow \infty} \frac{1}{k} \sum_{j=1}^{k}\left[\beta_{j}^{1} \mathbf{z}_{j}, \ldots,\right.\right. \\
\left.\left.\ldots, \beta_{j}^{l} \mathbf{z}_{j}\right] \eta_{j}\right\}
\end{gathered}
$$

onde $\mathbf{C}_{\mathbf{z x}}$ é não-singular e, conseqüentemente, a inversa existe. A partir do Lema 2, temos

$$
\text { p. } \lim _{k \rightarrow \infty} \tilde{\theta}_{k}=\left\{\text { p. } \lim _{k \rightarrow \infty} \mathbf{C}_{\mathbf{z x}}\right\}^{-1} \mathbf{0}
$$

Assim, o valor do erro paramétrico, no limite em probabilidade, é

$$
\text { p. } \lim _{k \rightarrow \infty} \tilde{\theta}=0
$$

e a estimação é assintoticamente não-polarizada, como desejado.

Em conseqüência desta análise, a definição do vetor $\left[\beta_{j}^{1} \mathbf{z}_{j}, \ldots, \beta_{j}^{l} \mathbf{z}_{j}\right]$ como vetor de variáveis instrumentais fuzzy ou simplesmente variáveis instrumentais fuzzy (VIF) é proposta. Claramente, com o uso do vetor VIF na forma sugerida, é possível eliminar a polarização assintótica e preservar a existência de uma solução. Porém, a eficiência estatística da solução é dependente do grau de correlação entre $\left[\beta_{j}^{1} \mathbf{z}_{j}, \ldots, \beta_{j}^{l} \mathbf{z}_{j}\right]$ e $\left[\gamma_{j}^{1} \mathbf{x}_{j}, \ldots, \gamma_{j}^{l} \mathbf{x}_{j}\right]$. Em particular, a menor variância da estimação ocorrerá quando $\mathbf{z}_{j}=\mathbf{x}_{j}$, isto é, quando $\mathbf{z}_{j}$ são iguais às variáveis do sistema livres de ruído, os quais estão indisponíveis na prática. Uma escolha eficiente de VIF seria baseada na seqüência de entrada atrasada

$$
\mathbf{z}_{j}=\left[u_{k-\tau}, \ldots, u_{k-\tau-n}, u_{k}, \ldots, u_{k-n}\right]^{T}
$$


onde $\tau$ é escolhido de modo que os elementos da matriz de covariância $\mathbf{C}_{\mathbf{z x}}$ são maximizados. Neste caso, o sinal de entrada é considerado excitante persistentemente, isto é, ele deve perturbar continuamente o sistema. Uma outra escolha de VIF seria baseada na seqüência de entrada-saída atrasada

$$
\mathbf{z}_{j}=\left[y_{k-1-d l}, \cdots, y_{k-n_{y}-d l}, u_{k-1-d l}, \cdots, u_{k-n_{u}-d l}\right]^{T}
$$

onde $d l$ é o atraso aplicado. Outra escolha de VIF seria baseada na sequiência de entrada-saída de um "modelo fuzzy auxiliar" com a mesma estrutura do modelo utilizado para identificar o sistema dinâmico original. Assim,

$$
\mathbf{z}_{j}=\left[\hat{y}_{k-1}, \cdots, \hat{y}_{k-n_{y}}, u_{k-1}, \cdots, u_{k-n_{u}}\right]^{T}
$$

onde $\hat{y}$ é a saída do modelo fuzzy auxiliar, e $u$ é a entrada do sistema dinâmico. A fórmula de inferência deste modelo auxiliar é dada por

$$
\begin{aligned}
& \hat{y}(k+1)=\beta_{1}\left(\mathbf{z}_{k}\right)\left[\alpha_{1,1} y(k)+\ldots+\alpha_{1, n y} y\left(k-n_{y}\right.\right. \\
& \left.+1)+\rho_{1,1} u(k)+\ldots+\rho_{1, n u} u\left(k-n_{u}+1\right)+\delta_{1}\right]+ \\
& +\beta_{2}\left(\mathbf{z}_{k}\right)\left[\alpha_{2,1} \hat{y}(k)+\ldots+\alpha_{2, n y} y\left(k-n_{y}+1\right)+\right. \\
& \left.+\rho_{2,1} u(k)+\ldots+\rho_{2, n u} u\left(k-n_{u}+1\right)+\delta_{2}\right]+\ldots \\
& +\beta_{l}\left(\mathbf{z}_{k}\right)\left[\alpha_{l, 1} \hat{y}(k)+\ldots+\alpha_{l, n y} y\left(k-n_{y}+1\right)+\right. \\
& \left.+\rho_{l, 1} u(k)+\ldots+\rho_{l, n u} u\left(k-n_{u}+1\right)+\delta_{l}\right]
\end{aligned}
$$

a qual é também linear nos parâmetros do conseqüente: $\alpha$, $\rho$ e $\delta$. Quanto mais próximos dos parâmetros reais, mas desconhecidos, (a, b, c) como em (11), mais correlacionados $\mathbf{z}_{k}$ e $\mathbf{x}_{k}$, e a estimação via VIF será próxima da ótima.

\subsection{Algoritmo de variável instrumental fuzzy}

Nesta seção é apresentada a formulação geral, off-line e online, do algoritmo de variável instrumental fuzzy, independente dos três métodos aqui apresentados em função de $\mathbf{z}_{j}$ : seqüência de entrada atrasada, seqüência de entradasaída atrasada, sequiência de entrada-saída de um modelo fuzzy auxiliar. Resultados computacionais bem como um estudo de caso à identificação de uma viga de alumínio (uma planta complexa, não-linear e variante com o tempo) cujo estudo serve como fundamento para aplicações em estruturas mecânicas de aeronaves e/ou veículos aeroespaciais podem ser vistos em ( ${ }^{b}$ Bottura and Serra, 2005).

\subsection{Esquema Off-line}

As equações normais VIF são formuladas como

$$
\begin{gathered}
\sum_{j=1}^{k}\left[\beta_{j}^{1} \mathbf{z}_{j}, \ldots, \beta_{j}^{l} \mathbf{z}_{j}\right]\left[\gamma_{j}^{1}\left(\mathbf{x}_{j}+\xi_{j}\right), \ldots, \gamma_{j}^{l}\left(\mathbf{x}_{j}+\right.\right. \\
\left.\left.+\xi_{j}\right)\right]^{T} \hat{\theta}_{k}-\sum_{j=1}^{k}\left[\beta_{j}^{1} \mathbf{z}_{j}, \ldots, \beta_{j}^{l} \mathbf{z}_{j}\right] y_{j}=0
\end{gathered}
$$

ou, $\operatorname{com} \zeta_{j}=\left[\beta_{j}^{1} \mathbf{z}_{j}, \ldots, \beta_{j}^{l} \mathbf{z}_{j}\right]$,

$$
\left[\sum_{j=1}^{k} \zeta_{j} \chi_{j}^{T}\right] \hat{\theta}_{k}-\sum_{j=1}^{k} \zeta_{j} y_{j}=0
$$

de modo que a estimação VIF é obtida por

$$
\begin{gathered}
\hat{\theta}_{k}=\left\{\sum _ { j = 1 } ^ { k } [ \beta _ { j } ^ { 1 } \mathbf { z } _ { j } , \ldots , \beta _ { j } ^ { l } \mathbf { z } _ { j } ] \left[\gamma_{j}^{1}\left(\mathbf{x}_{j}+\xi_{j}\right), \ldots, \gamma_{j}^{l}\left(\mathbf{x}_{j}+\right.\right.\right. \\
\left.\left.\left.\quad+\xi_{j}\right)\right]^{T}\right\}^{-1} \sum_{j=1}^{k}\left[\beta_{j}^{1} \mathbf{z}_{j}, \ldots, \beta_{j}^{l} \mathbf{z}_{j}\right] y_{j}
\end{gathered}
$$

e, na forma vetorial, o problema de interesse pode ser definido por:

$$
\hat{\theta}=\left(\boldsymbol{\Gamma}^{T} \boldsymbol{\Sigma}\right)^{-1} \boldsymbol{\Gamma}^{T} \mathbf{Y}
$$

onde $\Gamma^{T} \in \Re^{l\left(n_{y}+n_{u}+1\right) \times N}$ é a matriz de variável instrumental fuzzy extendida com linhas dadas por $\zeta_{j}, \Sigma \in$ $\Re^{N \times l\left(n_{y}+n_{u}+1\right)}$ é a matriz de dados extendida com linhas dadas por $\chi_{j}$ e $\mathbf{Y} \in \Re^{N \times 1}$ é o vetor de saída e $\hat{\theta} \in$ $\Re^{l\left(n_{y}+n_{u}+1\right) \times 1}$ é o vetor de parâmetros.

\subsection{Esquema online}

A estimação VIF em (33) pode ser da forma

$$
\hat{\theta}_{k}=\mathbf{P}_{k} \mathbf{b}_{k}
$$

onde

$$
\begin{gathered}
\mathbf{P}_{k}=\left\{\sum _ { j = 1 } ^ { k } [ \beta _ { j } ^ { 1 } \mathbf { z } _ { j } , \ldots , \beta _ { j } ^ { l } \mathbf { z } _ { j } ] \left[\gamma_{j}^{1}\left(\mathbf{x}_{j}+\xi_{j}\right), \ldots, \gamma_{j}^{l}\left(\mathbf{x}_{j}+\right.\right.\right. \\
\left.\left.\left.+\xi_{j}\right)\right]^{T}\right\}^{-1}
\end{gathered}
$$

e

$$
\mathbf{b}_{k}=\sum_{j=1}^{k}\left[\beta_{j}^{1} \mathbf{z}_{j}, \ldots, \beta_{j}^{l} \mathbf{z}_{j}\right] y_{j}
$$

a qual pode ser expressa como

$$
\mathbf{P}_{k}^{-1}=\mathbf{P}_{k-1}^{-1}+\left[\beta_{k}^{1} \mathbf{z}_{k}, \ldots, \beta_{k}^{l} \mathbf{z}_{k}\right]\left[\gamma_{k}^{1}\left(\mathbf{x}_{k}+\xi_{k}\right), \ldots,\right.
$$




$$
\left.\ldots, \gamma_{k}^{l}\left(\mathbf{x}_{k}+\xi_{k}\right)\right]^{T}
$$

$\mathrm{e}$

$$
\mathbf{b}_{k}=\mathbf{b}_{k-1}+\left[\beta_{k}^{1} \mathbf{z}_{k}, \ldots, \beta_{k}^{l} \mathbf{z}_{k}\right] y_{k}
$$

respectivamente. Pré-multiplicando (36) por $\mathbf{P}_{k}$ e pósmultiplicando por $\mathbf{P}_{k-1}$ resulta

$$
\begin{gathered}
\mathbf{P}_{k-1}=\mathbf{P}_{k}+\mathbf{P}_{k}\left[\beta_{k}^{1} \mathbf{z}_{k}, \ldots, \beta_{k}^{l} \mathbf{z}_{k}\right]\left[\gamma_{k}^{1}\left(\mathbf{x}_{k}+\xi_{k}\right), \ldots,\right. \\
\left.\ldots, \gamma_{k}^{l}\left(\mathbf{x}_{k}+\xi_{k}\right)\right]^{T} \mathbf{P}_{k-1}
\end{gathered}
$$

e pós-multiplicando pelo vetor VIF, resulta

$$
\begin{gathered}
\mathbf{P}_{k-1}\left[\beta_{k}^{1} \mathbf{z}_{k}, \ldots, \beta_{k}^{l} \mathbf{z}_{k}\right]=\mathbf{P}_{k}\left[\beta_{k}^{1} \mathbf{z}_{k}, \ldots, \beta_{k}^{l} \mathbf{z}_{k}\right]\{1+ \\
{\left[\gamma_{k}^{1}\left(\mathbf{x}_{k}+\xi_{k}\right), \ldots, \gamma_{k}^{l}\left(\mathbf{x}_{k}+\xi_{k}\right)\right]^{T} \mathbf{P}_{k-1}\left[\beta_{k}^{1} \mathbf{z}_{k}, \ldots,\right.} \\
\left.\left.\ldots, \beta_{k}^{l} \mathbf{z}_{k}\right]\right\}
\end{gathered}
$$

Então, pós-multiplicando por $\left\{1+\left[\gamma_{k}^{1}\left(\mathbf{x}_{k}+\xi_{k}\right), \ldots, \gamma_{k}^{l}\left(\mathbf{x}_{k}+\right.\right.\right.$ $\left.\left.\left.\xi_{k}\right)\right]^{T} \mathbf{P}_{k-1}\left[\beta_{k}^{1} \mathbf{z}_{k}, \ldots, \beta_{k}^{l} \mathbf{z}_{k}\right]\right\}^{-1}\left[\gamma_{k}^{1}\left(\mathbf{x}_{k}+\xi_{k}\right), \ldots, \gamma_{k}^{l}\left(\mathbf{x}_{k}+\right.\right.$ $\left.\left.\xi_{k}\right)\right]^{T} \mathbf{P}_{k-1}$, tem-se

$$
\begin{gathered}
\mathbf{P}_{k-1}\left[\beta_{k}^{1} \mathbf{z}_{k}, \ldots, \beta_{k}^{l} \mathbf{z}_{k}\right]\left\{1+\left[\gamma_{k}^{1}\left(\mathbf{x}_{k}+\xi_{k}\right), \ldots, \gamma_{k}^{l}\left(\mathbf{x}_{k}+\right.\right.\right. \\
\left.\left.\left.\xi_{k}\right)\right]^{T} \mathbf{P}_{k-1}\left[\beta_{k}^{1} \mathbf{z}_{k}, \ldots, \beta_{k}^{l} \mathbf{z}_{k}\right]\right\}^{-1}\left[\gamma_{k}^{1}\left(\mathbf{x}_{k}+\xi_{k}\right), \ldots,\right. \\
\left.\gamma_{k}^{l}\left(\mathbf{x}_{k}+\xi_{k}\right)\right]^{T} \mathbf{P}_{k-1}=\mathbf{P}_{k}\left[\beta_{k}^{1} \mathbf{z}_{k}, \ldots, \beta_{k}^{l} \mathbf{z}_{k}\right]\left[\gamma _ { k } ^ { 1 } \left(\mathbf{x}_{k}+\right.\right. \\
\left.\left.\xi_{k}\right), \ldots, \gamma_{k}^{l}\left(\mathbf{x}_{k}+\xi_{k}\right)\right]^{T} \mathbf{P}_{k-1}
\end{gathered}
$$

Substituindo (38) em (39), resulta

$$
\begin{gathered}
\mathbf{P}_{k}=\mathbf{P}_{k-1}-\mathbf{P}_{k-1}\left[\beta_{k}^{1} \mathbf{z}_{k}, \ldots, \beta_{k}^{l} \mathbf{z}_{k}\right]\left\{1+\left[\gamma _ { k } ^ { 1 } \left(\mathbf{x}_{k}+\right.\right.\right. \\
\left.\left.\left.+\xi_{k}\right), \ldots, \gamma_{k}^{l}\left(\mathbf{x}_{k}+\xi_{k}\right)\right]^{T} \mathbf{P}_{k-1}\left[\beta_{k}^{1} \mathbf{z}_{k}, \ldots, \beta_{k}^{l} \mathbf{z}_{k}\right]\right\}^{-1} \\
{\left[\gamma_{k}^{1}\left(\mathbf{x}_{k}+\xi_{k}\right), \ldots, \gamma_{k}^{l}\left(\mathbf{x}_{k}+\xi_{k}\right)\right]^{T} \mathbf{P}_{k-1}}
\end{gathered}
$$

Substituindo (40) e (37) em (35), a equação recursiva para os parâmetros do conseqüente será:

$$
\begin{gathered}
\hat{\theta}_{k}=\left\{\mathbf{P}_{k-1}-\mathbf{P}_{k-1}\left[\beta_{k}^{1} \mathbf{z}_{k}, \ldots, \beta_{j}^{l} \mathbf{z}_{k}\right]\left\{1+\left[\gamma _ { k } ^ { 1 } \left(\mathbf{x}_{k}+\right.\right.\right.\right. \\
\left.\left.\left.+\xi_{k}\right), \ldots, \gamma_{k}^{l}\left(\mathbf{x}_{k}+\xi_{k}\right)\right]^{T} \mathbf{P}_{k-1}\left[\beta_{k}^{1} \mathbf{z}_{k}, \ldots, \beta_{k}^{l} \mathbf{z}_{k}\right]\right\}^{-1} \\
\left.\left[\gamma_{k}^{1}\left(\mathbf{x}_{k}+\xi_{k}\right), \ldots, \gamma_{k}^{l}\left(\mathbf{x}_{k}+\xi_{k}\right)\right]^{T} \mathbf{P}_{k-1}\right\}\left\{\mathbf{b}_{k-1}+\right. \\
\left.\left[\beta_{k}^{1} \mathbf{z}_{k}, \ldots, \beta_{k}^{l} \mathbf{z}_{k}\right] y_{k}\right\}
\end{gathered}
$$

de maneira que, finalmente

$$
\begin{gathered}
\hat{\theta}_{k}=\hat{\theta}_{k-1}-\mathbf{K}_{k}\left\{\left[\gamma_{j}^{1}\left(\mathbf{x}_{k}+\xi_{k}\right), \ldots, \gamma_{k}^{l}\left(\mathbf{x}_{k}+\right.\right.\right. \\
\left.\left.\left.+\xi_{k}\right)\right]^{T} \hat{\theta}_{k-1}-y_{k}\right\}
\end{gathered}
$$

onde

$$
\begin{gathered}
\mathbf{K}_{k}=\mathbf{P}_{k-1}\left[\beta_{k}^{1} \mathbf{z}_{k}, \ldots, \beta_{k}^{l} \mathbf{z}_{k}\right]\left\{1+\left[\gamma_{k}^{1}\left(\mathbf{x}_{k}+\xi_{k}\right), \ldots\right.\right. \\
\left.\left.\ldots, \gamma_{k}^{l}\left(\mathbf{x}_{k}+\xi_{k}\right)\right]^{T} \mathbf{P}_{k-1}\left[\beta_{k}^{1} \mathbf{z}_{k}, \ldots, \beta_{k}^{l} \mathbf{z}_{k}\right]\right\}^{-1}
\end{gathered}
$$

\section{RESULTADOS COMPUTACIONAIS}

\subsection{Exemplo: planta não-linear}

A planta a ser identificada consiste em um sistema altamente não-linear de segunda ordem

$$
y(k+1)=\frac{y(k) y(k-1)(y(k)+2.5)}{1+y(k)^{2}+y(k-1)^{2}}+u(k)+e(k)
$$

que é uma referência muito utilizada em modelagem fuzzy, onde $y(k)$ é a saída e $u(k)=\sin \left(\frac{2 \pi k}{25}\right)$ é a entrada aplicada. Neste caso $e(k)$ é um ruído branco com média zero e variância $\sigma^{2}$. O modelo TS tem duas entradas $y(k)$ e $y(k-1)$ e uma saída $y(k+1)$, e os parâmetros do antecedente na base de regras do modelo fuzzy são definidos pelo método de agrupamento ECM (Evolving Clustering Method) proposto em (Kasabov and Song, 2002). O modelo é composto de regras da forma:

$$
\begin{gathered}
R^{i}: \operatorname{IF} y(k) \text { is } F_{1}^{i} \text { AND } y(k-1) \text { is } F_{2}^{i} \text { THEN } \\
\hat{y}_{i}(k+1)=a_{i, 1} y(k)+a_{i, 2} y(k-1)+b_{i, 1} u(k)+c_{i}
\end{gathered}
$$

onde $F_{1,2}^{i}$ são conjuntos fuzzy com funções gaussianas. Conjuntos de dados experimentais de $N$ pontos cada são criados a partir de (43), com $\sigma^{2} \in[0,0.23]$. Isto quer dizer que o ruído aplicado tem valores entre 0 e $\pm 30 \%$ do valor da saída nominal, que é uma percentagem de ruído aceitável na prática. Estes conjuntos de dados são apresentados ao algoritmo proposto, para a obtenção de um modelo fuzzy IV, e a um algoritmo baseado em LS, para a obtenção de um modelo fuzzy LS. Os modelos são obtidos pelas seguintes abordagens:

- Abordagem global : Nesta abordagem, todos os parâmetros do conseqüente são estimados simultaneamente, minimizando o critério:

$$
\hat{\boldsymbol{\Theta}}=\arg \min \|\mathbf{S} \Theta-\mathbf{b}\|_{2}^{2}
$$

com $\mathbf{S}=\boldsymbol{\Gamma}^{T} \mathbf{W} \boldsymbol{\Sigma}$ e $\mathbf{b}=\boldsymbol{\Gamma}^{T} \mathbf{W} \mathbf{Y}$ para o algoritmo proposto, onde $\boldsymbol{\Gamma}$ é a matriz de variável instrumental estendida e $\mathbf{W}=\operatorname{diag}\left(\lambda^{n-1}, \lambda^{n-2}, \ldots, 1\right) \in \Re^{N \times N}$, com $0<\lambda<1$; e $\mathbf{S}=\boldsymbol{\Sigma}^{T} \boldsymbol{\Sigma}$ e $\mathbf{b}=\boldsymbol{\Sigma}^{T} \mathbf{Y}$ para o algoritmo baseado no método de mínimos quadrados, onde $\Sigma$ é a matriz de dados estendida. O escalar $\lambda$, fator de esquecimento, é usado para atribuir menos peso aos dados passados.

- Abordagem local : Nesta abordagem os parâmetros do conseqüente são estimados para cada regra $i$, independentemente uns dos outros, minimizando o conjunto de critérios locais ponderados $(i=$ $1,2, \ldots, l)$ :

$$
\hat{\boldsymbol{\Theta}}_{i}=\arg \min \left\|\mathbf{S}_{i} \boldsymbol{\Theta}_{i}-\mathbf{b}_{i}\right\|_{2}^{2}
$$


com $\mathbf{S}_{i}=\mathbf{Z}^{T} \boldsymbol{\Psi}_{i} \mathbf{X}$ e $\mathbf{b}_{i}=\mathbf{Z}^{T} \boldsymbol{\Psi}_{i} \mathbf{Y}$ para o algoritmo proposto, onde $\mathbf{Z}$ é a matriz de variável instrumental e $\boldsymbol{\Psi}_{i}$ é a matriz de graus de ativação normalizados de cada regra fuzzy; e $\mathbf{S}_{i}=\mathbf{X}^{T} \boldsymbol{\Psi}_{i} \mathbf{X}$ e $\mathbf{b}_{i}=\mathbf{X}^{T} \boldsymbol{\Psi}_{i} \mathbf{Y}$ para o algoritmo baseado no método de mínimos quadrados, onde $\mathbf{X}$ é a matriz de dados.

A influência do ruído é analisada de acordo com a diferença antre as saídas dos modelos fuzzy obtidos a partir dos dados ruidosos, e a saída da planta sem ruído. Os parâmetros do antecedente e a estrutura dos modelos fuzzy são as mesmas nos experimentos, enquanto os parâmetros do conseqüente são obtidos pelo método proposto e pelo método LS. Assim, os resultados obtidos são devidos a estes algoritmos e conclusões precisas serão obtidas sobre o desempenho do algoritmo proposto na presença de ruído. Dois critérios são aplicados para validação dos modelos fuzzy obtidos : VAF (Variance Accounted For)

$$
\mathbf{V A F}(\%)=100 \times\left[1-\frac{\operatorname{var}(\mathbf{Y}-\hat{\mathbf{Y}})}{\operatorname{var}(\mathbf{Y})}\right]
$$

onde $\mathbf{Y}$ é a saída nominal da planta, $\hat{\mathbf{Y}}$ é a saída do modelo fuzzy e var é a variância do sinal, e MSE (Mean Square Error)

$$
\text { MSE }=\frac{1}{N} \sum_{k=1}^{N}(y(k)-\hat{y}(k))^{2}
$$

onde $y(k)$ é a saída nominal da planta, $\hat{y}(k)$ é a saída do modelo fuzzy, $N$ é o número de pontos. Uma vez obtidos estes valores, uma análise comparativa é estabelecida entre o algoritmo proposto, baseado em IV, e o algoritmo baseado em LS, de acordo com as abordagens apresentadas acima. A Fig. 2 mostra os desempenhos dos modelos obtidos off-line de acordo com (45) e (46). Para estes resultados, o número de pontos foi 500, o algoritmo proposto usou um fator de esquecimento $\lambda$ igual a 0.99 ; o número de regras foi 4, a estrutura foi apresentada em (44) e os parâmetros do antecedente foram obtidos pelo método ECM para ambos os algoritmos. O fator de esquecimento bem como o número de regras foram escolhidos levando-se em consideração a velocidade de estimação paramétrica bem como a simplicidade do modelo fuzzy. A partir da Fig. 2 pode-se ver, claramente, que o algoritmo proposto apresenta melhor desempenho que o algoritmo baseado em LS nas duas abordagens, sendo mais robusto ao ruído. Isto é devido à matriz de variável instrumental escolhida baseada na seqüência de entrada e saída atrasada, com $l d=1$, satisfazendo as condições dos Lemas e Teorema propostos tão bem quanto possível. Na abordagem global, para baixa variância do ruído, ambos algoritmos apresentaram desempenhos similares com VAF e MSE de $99.50 \%$ e 0.0071 para o algoritmo proposto e de $99.56 \%$ e 0.0027 para o
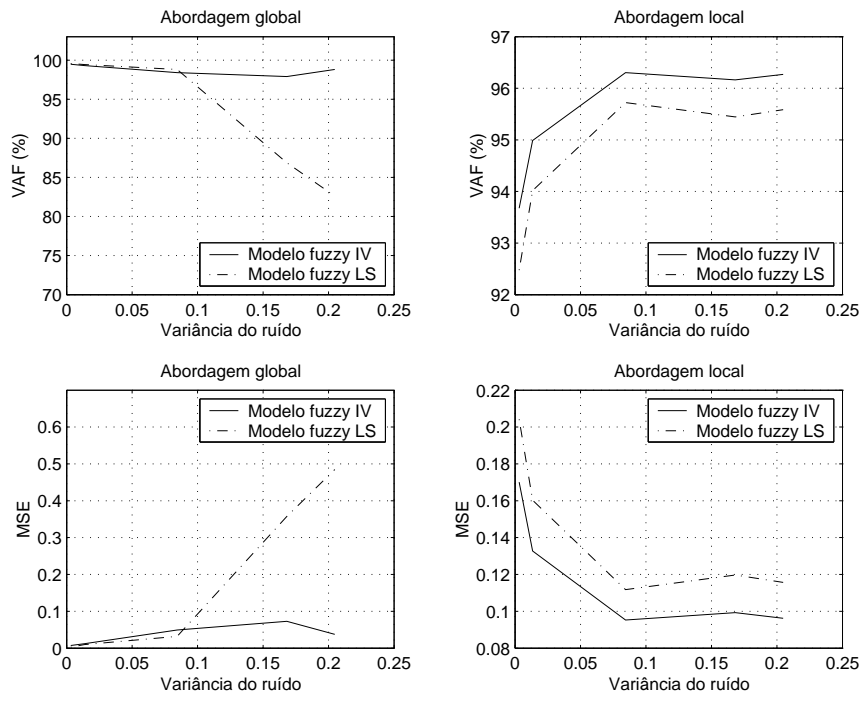

Figura 2: Análise comparativa

algoritmo baseado em LS, respectivamente. Porém, quando a variância do ruído aumenta, a matriz de variável instrumental escolhida satisfaz os Lemas e Teorema propostos, o que, conseqüentemente, torna o algoritmo proposto mais robusto ao ruído com VAF e MSE de $98.81 \%$ e 0.0375 , enquanto que o algoritmo baseado em LS apresentou VAF e MSE de $82.61 \%$ e 0.4847 , respectivamente. Uma análise similar pode ser feita com a abordagem local: aumentando a variância do ruído, ambos algoritmos apresentam bom desempenho onde os valores de VAF e MSE aumentam também. Isto é devido à propriedade politópica (ver seção 2), onde os modelos obtidos podem representar aproximações locais com maior flexibilidade. O algoritmo proposto apresentou valores de VAF e MSE de $93.70 \%$ e 0.1701 para o pior caso e de $96.3 \%$ e 0.0962 para o melhor caso. O algoritmo baseado em LS apresentou valores de VAF e MSE de $92.4 \%$ e 0.2042 para o pior caso e de $95.5 \%$ e 0.1157 para o melhor caso. A Fig. 3 mostra parte dos dados de saída ruidosos para, $\sigma^{2}=0.24$, bem como as saídas dos modelos fuzzy para ambos algoritmos e a saída da planta livre de ruído para ambas abordagens objetivando a validação dos modelos. Claramente, pode ser observada a precisão da estimação da saída para o método proposto para ambas abordagens. Para a abordagem global, os valores de VAF e MSE foram de $96.5 \%$ e 0.09 para o método proposto e de $81.4 \%$ e 0.52 para o algoritmo baseado em LS, respectivamente. Para a abordagem local, os valores de VAF e MSE foram de $96.0 \%$ e 0.109 para o método proposto e de $95.5 \%$ e 0.1187 para o algoritmo baseado em LS, respectivamente. Para a versão recursiva implementada de acordo com a seção 4.3 , a análise de desempenho do algoritmo proposto é feita para a seguinte 

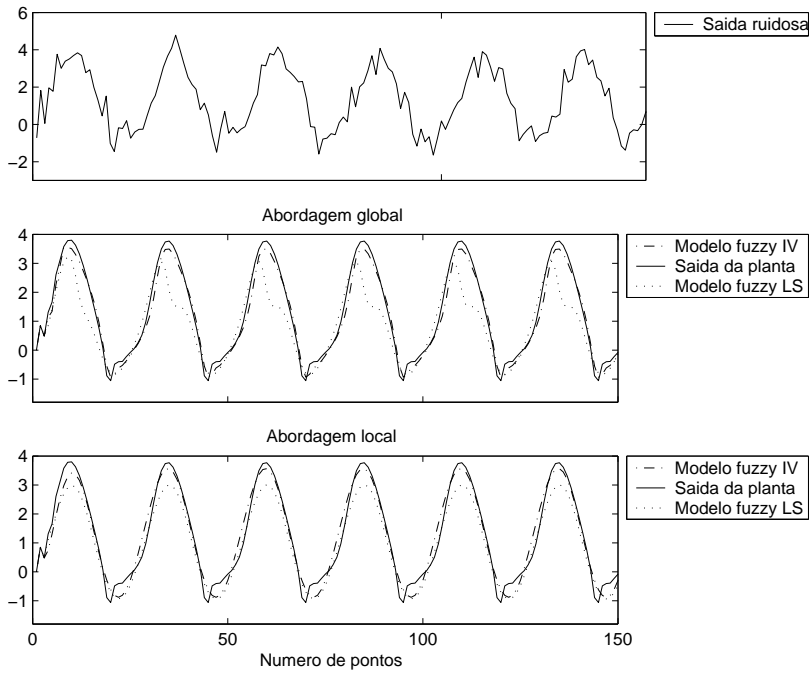

Figura 3: Validação dos modelos

planta não-linear

$y(k+1)=\frac{y(k) y(k-1)(y(k)+p r m t(k))}{1+y(k)^{2}+y(k-1)^{2}}+u(k)+e(k)$

onde $\operatorname{prmt}(k)=2.5+0.1 \sin \left(\frac{2 \pi k}{45}\right)$. A Fig. 4 mostra o rastreamento da saída da planta não-linear variante no tempo em um ambiente ruidoso com $\sigma^{2}=0.01$. Nesta aplicação, 25 pontos foram usados para fazer a estimação inicial e $\lambda=0.99$. Como esperado, o método proposto fornece uma estimação da saída com alta precisão, uma estimação consistente e não-polarizada dos parâmetros fuzzy e, conseqüentemente, alta velocidade de convergência
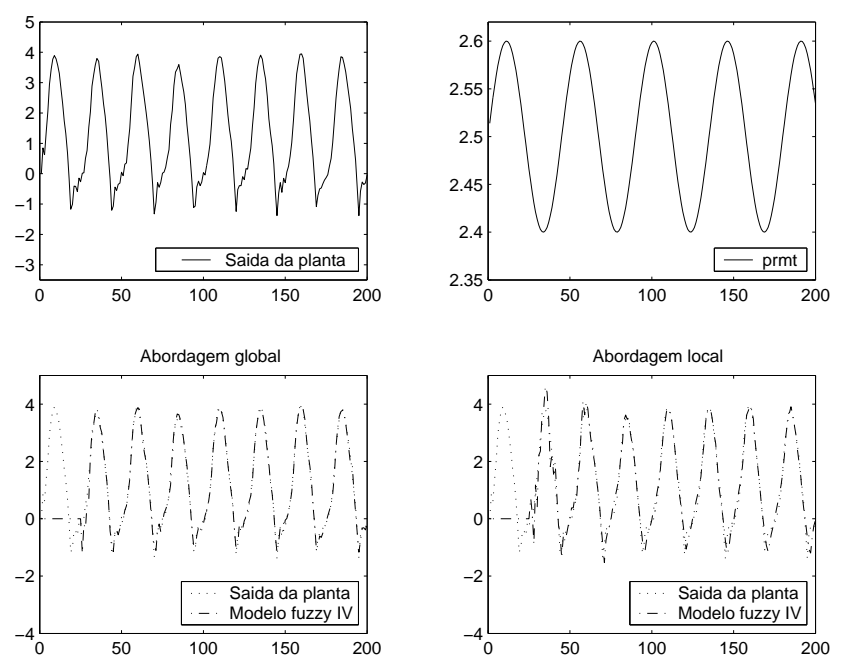

Figura 4: Rastreamento da saída da planta não-linear variante no tempo dos modelos fuzzy para seguir o comportamento variante no tempo da planta em um ambiente ruidoso. Estas características são muito importantes em aplicações ao projeto de controle adaptativo.

\subsection{Estudo de caso: Viga livre-livre}

Esta seção estaremos dedicando à identificação de uma viga de alumínio, uma planta complexa, não-linear e variante com o tempo, cujas dimensões são: 1100x32x3 $\mathrm{mm}$ cujo estudo serve como fundamento para aplicações em estruturas mecânicas de aeronaves e/ou veículos aeroespaciais. A estrutura geral do experimento é do tipo livre-livre, ou seja, nenhuma das suas extremidades está fixa e a viga está suspensa por fios de nilon conforme mostra a Fig.5.

Esta viga faz parte do laboratório de Vibroacústica do Departamento de Mecânica Computacional na Faculdade de Engenharia Mecânica da UNICAMP. Vários estudos têm sido aplicados com o objetivo de se obter um modelo suficientemente preciso para fins de controle ativo de vibração. Foram utilizados 49152 pontos num intervalo de $49,152 \mathrm{~s}$, que implica num intervalo de amostragem de $1 \mathrm{~ms}$.

Um sinal senoidal com frequiência variando de 1 a 125 $\mathrm{Hz}$ foi utilizado como entrada, em três repetições, e a vibração (leitura a partir de um micro-acelerômetro) da viga de alumínio como a saída ruidosa. O conjunto de dados de entrada-saída durante todo o experimento são mostrados na Fig. 6 (a) e (c), enquanto que os primeiros $20 \mathrm{~ms}$ dos dados, para fins de ilustração, são apresentados na Fig. 6 (b) e (d). Este tipo de sinal de entrada apresenta boas propriedades estatísticas e é de grande interesse prático pois permite que os modos da planta, na varredura da frequiência, sejam excitados e um modelo mais preciso seja obtido. Foram utilizados os primeiros 24576 pontos para estimação paramétrica e os próximos 24576 , o que equivale à segunda metade do conjunto de dados, para a validação. Neste caso, as variáveis instrumentais fuzzy são as variáveis linguísticas $y_{k}$ e $y_{k-1}$ filtradas, geradas automaticamente a partir do modelo auxiliar fuzzy, e usada para criar as funções de

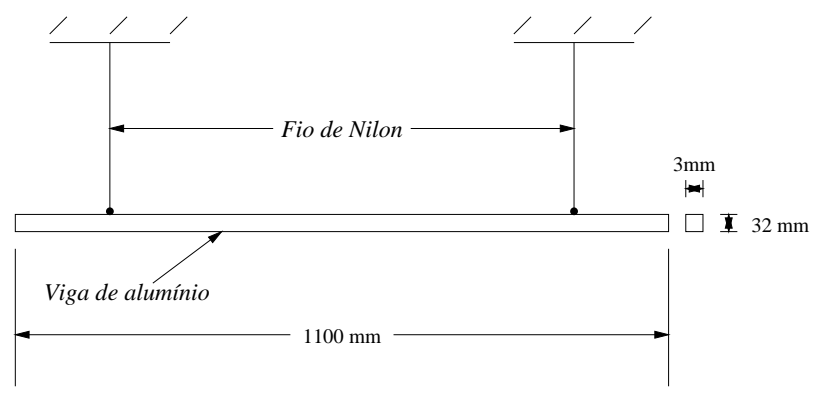

Figura 5: Viga de alumínio livre-livre 

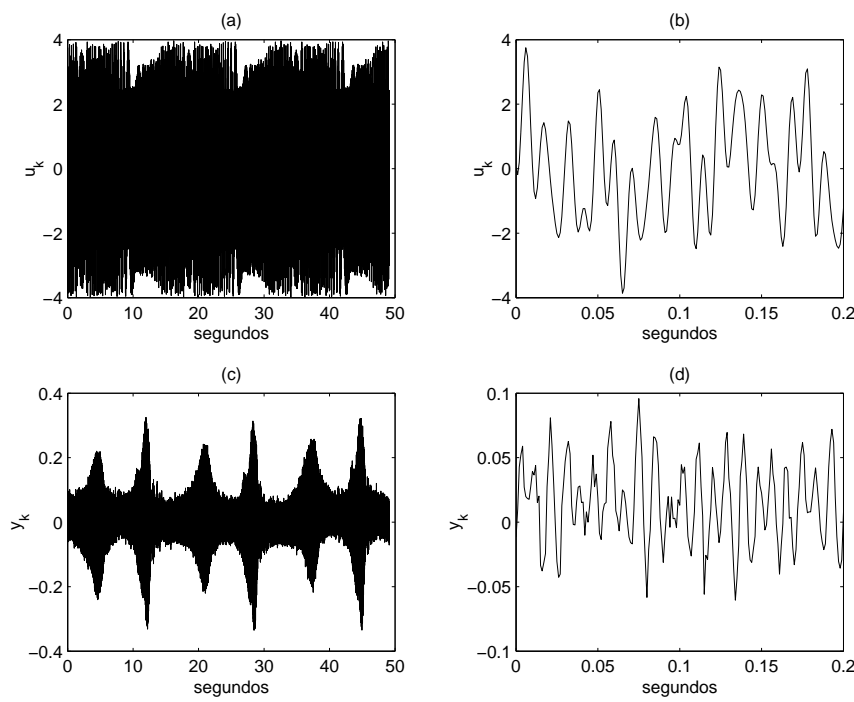

Figura 6: Teste dinâmico em uma viga livre-livre. Sinais de (a-b) entrada e (c-d) saída resultantes de um teste experimental. A entrada é o sinal que comanda a viga e a saída é a vibração.

pertinência bem como a matriz de variável instrumental fuzzy para estimação ótima dos parâmetros do conseqüente. A Fig. 7 mostra as partições das variáveis linguísticas do modelo fuzzy obtido. A Fig. 8 mostra os parâmetros estimados usando o algoritmo de variável instrumental fuzzy recursivo, e na Fig. 9 a matriz de covariância $P_{k}$ na última recursão. Esta matriz apresenta valores elevados na diagonal principal, implicando num melhor ajuste dos parâmetros estimados a cada recursão; e é simétrica, o que representa a consistência e estabilidade do algoritmo proposto para estimação dos parâmetros do conseqüente. A base de regras do modelo fuzzy obtido na última recursão é da seguinte forma:

$$
R^{1}: \text { SE } \hat{y}_{k} \text { é } F_{1} \text { E } \hat{y}_{k-1} \text { é } G_{1} \text { ENTÃO }
$$

$$
\hat{y}_{k+1}=6.3565 \hat{y}_{k}-3.4363 \hat{y}_{k-1}-0.0025 u_{k}-0.6552
$$

$$
R^{2}: \text { SE } \hat{y}_{k} \text { é } F_{1} \text { E } \hat{y}_{k-1} \text { é } G_{2} \text { ENTÃO }
$$

$$
\hat{y}_{k+1}=6.0951 \hat{y}_{k}-5.2626 \hat{y}_{k-1}+0.0067 u_{k}-2.0973
$$

$$
R^{3}: \text { SE } \hat{y}_{k} \text { é } F_{2} \text { E } \hat{y}_{k-1} \text { é } G_{1} \text { ENTÃO }
$$

$$
\hat{y}_{k+1}=7.3567 \hat{y}_{k}-5.4944 \hat{y}_{k-1}+0.0037 u_{k}+3.1799
$$

$$
R^{4}: \text { SE } \hat{y}_{k} \text { é } F_{2} \text { E } \hat{y}_{k+1} \text { é } G_{2} \text { ENTÃO }
$$
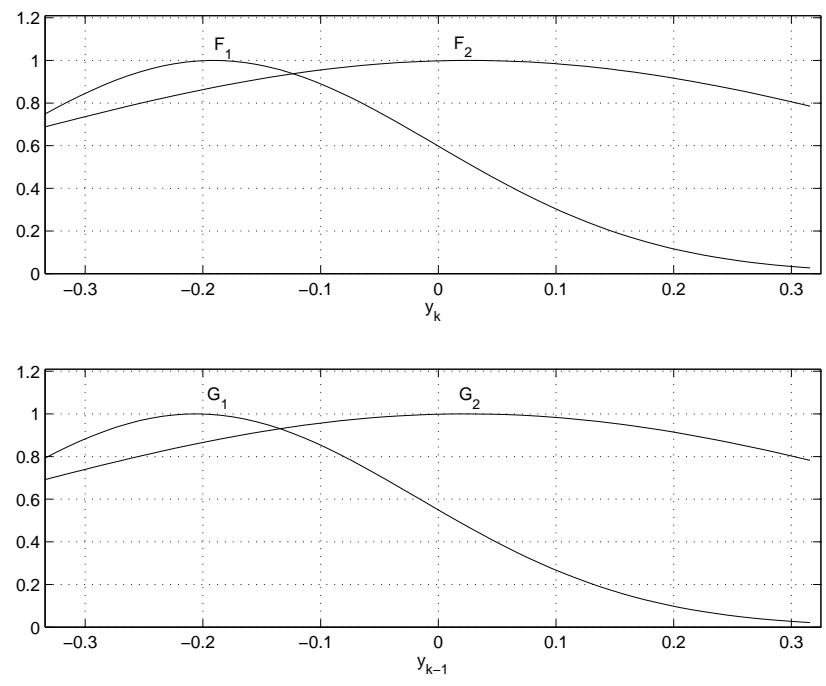

Figura 7: Partição das variáveis linguísticas do modelo fuzzy identificado.
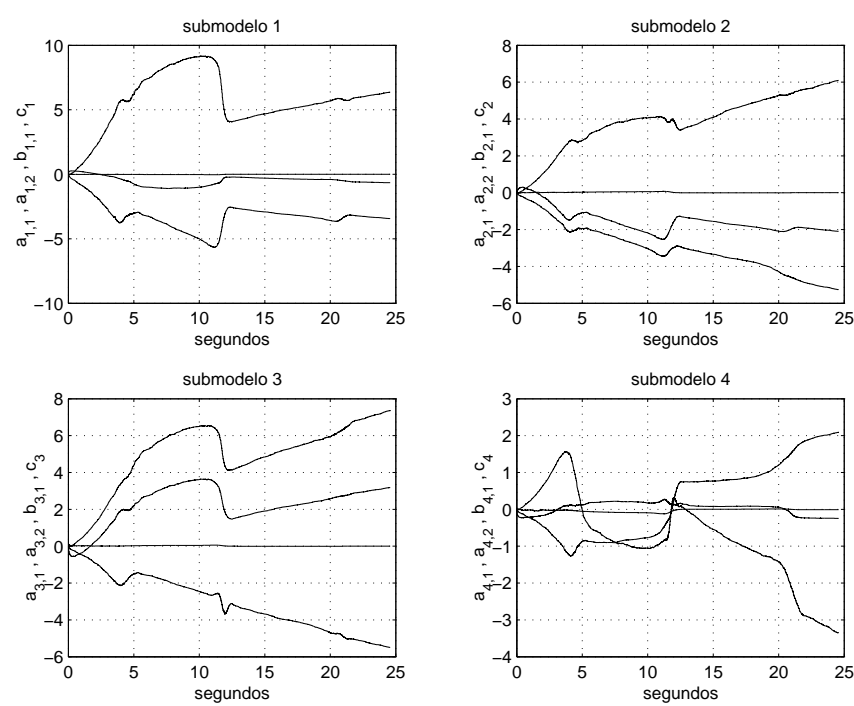

Figura 8: Estimação recursiva dos parâmetros do modelo fuzzy para a viga de alumínio.

$$
\hat{y}_{k+1}=-3.3510 \hat{y}_{k}+2.0907 \hat{y}_{k-1}-0.0112 u_{k}-0.2468
$$

O erro de estimação, o qual representa o ruído a partir dos componentes eletrônicos incluindo o atuador, sensor, sistema de aquisição de dados e amplificador de sinais, apresentou um valor quadrático médio MSE (Mean Square Error) de $4.2808 \times 10^{-4}$. A Fig. 10 mostra a validação do modelo fuzzy obtido de acordo com a curva da função de resposta em freqüência (FRF) (Juang, 1994) da viga de alumínio. A complexidade consiste nas três freqüências naturais da viga 


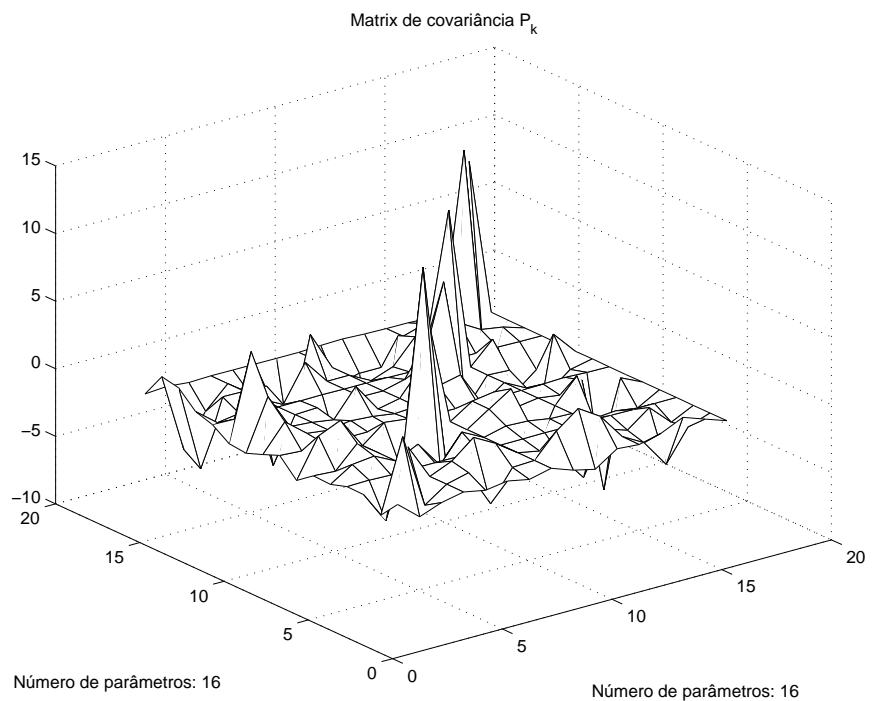

Figura 9: Matrix de covariância $P_{k}$.
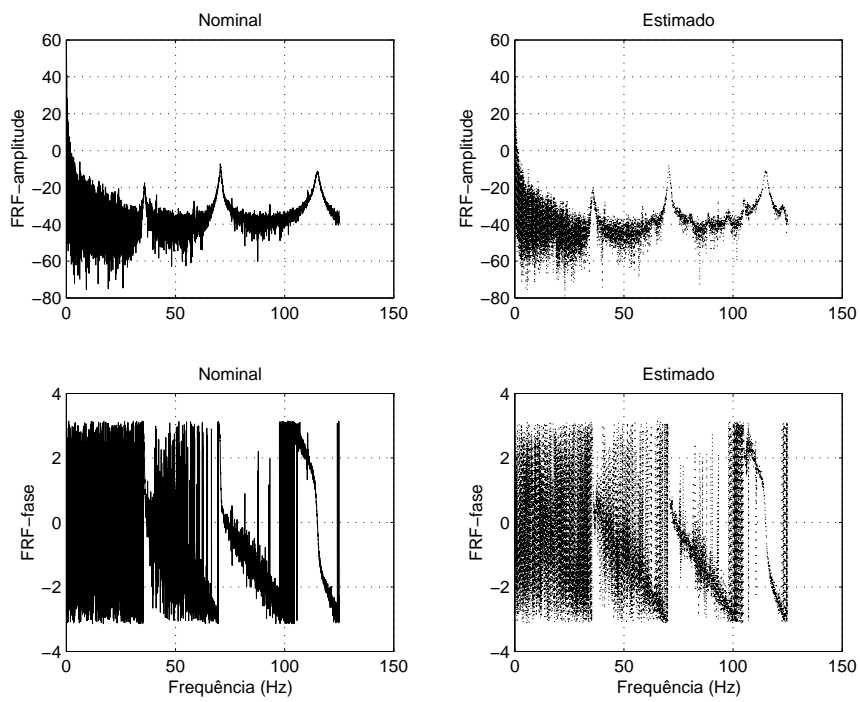

Figura 10: Validação do modelo.

de alumínio representadas pelos picos em $35 \mathrm{~Hz}, 70 \mathrm{~Hz}$ e 120 $\mathrm{Hz}$, respectivamente. Claramente, o modelo fuzzy obtido é suficientemente preciso na faixa de interesse do experimento e pode ser usado eficientemente no projeto de controle ativo de vibração.

\section{CONCLUSÃO}

A partir do conceito de variável instrumental fuzzy proposto em ( $\left({ }^{a}\right.$ Bottura and Serra, 2005), métodos de variável instrumental fuzzy para identificação de sistemas em ambientes ruidosos são propostos teoricamente. Condições de convergência para identificação em ambiente ruidoso em um contexto fuzzy foram estudadas. Um estudo de caso à identificação de uma viga de alumínio (uma planta complexa, não-linear e variante com o tempo) cujo estudo serve como fundamento para aplicações em estruturas mecânicas de aeronaves e/ou veículos aeroespaciais, foi apresentado. Resultados de simulação confirmaram a eficiência do método proposto que tem as seguintes características: a matriz de variável instrumental escolhida satisfaz os Lemas e Teoremas propostos, tornando o algoritmo proposto robusto ao ruído; estimação não-polarizada e consistente dos parâmetros fuzzy; convergência dos modelos fuzzy ao comportamento variante no tempo da planta não-linear; aplicabilidade ao projeto de controle adaptativo. Para trabalho futuro, a generalização do método proposto para plantas MIMO bem como sua aplicação ao projeto de controle adaptativo devem ser considerados.

\section{AGRADECIMENTOS}

Os autores gostariam de agradecer à CAPES e ao CNPq pelo apoio financeiro a este trabalho.

\section{REFERÊNCIAS}

Bottura, C.P. and Serra, G.L.O. (2004). An Algorithm for Fuzzy Identification of Nonlinear Discrete-Time Systems, $43^{\text {rd }}$ IEEE Conference on Decision and Control CDC'04, Atlantis(Paradise Island)Bahamas, December, pp. 5421-5426.

${ }^{a}$ Bottura, C.P. and Serra, G.L.O. (2005). Fuzzy Instrumental Variable Concept and Identification Algorithm, IEEE International Conference on Fuzzy Systems FUZZYIEEE'05, Nevada-EUA, May 22-25.

${ }^{b}$ Bottura, C.P. and Serra, G.L.O. (2005). Variável Instrumental Fuzzy, IV Congresso Temático de Dinâmica e Controle DINCON'05, Baurú-SP, Junho 610.

Brown, M. and Harris, C. (1994). Neurofuzzy Adaptive Modelling and Control, Prentice Hall.

Goodwin, G.C. and Payne, R.L. (1977). Dynamic System Identification: Experiment Design and Data Analysis, Academic Press.

Juang, Jer-Nan. (1994). Applied System Identification, Prentice Hall.

Kasabov, N.K. and Song, Q. (2002). DENFIS: Dynamic Evolving Neural-Fuzzy Inference System and Its Application for Time-Series Prediction,IEEE Transactions on Fuzzy Systems, Vol. 10, No 2, pp. 144154. 
Kukolj, D. and Levi, E. (2004). Identification of Complex Systems Based on Neural and Takagi-Sugeno Fuzzy Model,IEEE Transactions on Systems, Man and Cybernetics, Vol. 34, No 1, pp. 272-282.

Kuma, M., Stoll, R. and Stoll, N. (2006). A Robust Design Criterion for Interpretable Fuzzy Models with Uncertain Data ,IEEE Transactions on Fuzzy Systems, Vol. 14, No 2, pp. 314-328.

Ljung, L. (1999). System Identification: Theory for user, 2nd ed., Prentice Hall.

Ljung, L. and Söderström, T. (1987). Theory and Practice of Recursive Identification, MIT PRESS.

Papadakis, S.E. and Theocaris, J.B. (2002). A GAbased Fuzzy Modeling Approach for Generating TSK Models, Fuzzy Sets and Systems, Vol. 131, No 2, pp. 121-152.

Serra, G.L.O. and Bottura, C.P. (2006). Multiobjective Evolution Based Fuzzy PI Controller Design For Nonlinear Systems, IFAC Engineering Applications of Artificial Intelligence, Vol. 19, No. 2, pp. 157-167.

Serra, G.L.O. (2005). Propostas de Metodologias para Identificação e Controle Inteligentes, Tese de Doutorado, Laboratório de Controle e Sistemas Inteligentes, UNICAMP/FEEC/DMCSI, Campinas-SP.

Skrjanc, L., Blazic, S. and Agamennoni, O. (2005). Interval Fuzzy Model Identification Using $l_{\infty}$-Norm ,IEEE Transactions on Fuzzy Systems, Vol. 13, No 5, pp. 561568.

${ }^{a}$ Söderström, T. and Stoica, P.G. (1989). System Identification, Prentice Hall.

${ }^{b}$ Söderström, T. and Stoica, P.G. (1983). Instrumental Variable Methods for System Identification, Lecture Notes in Control and Information Sciences, SpringerVerlag.

Takagi, T. and Sugeno, M. (1985). Fuzzy Identification of Systems and Its Application to Modeling and Control, IEEE Transations on Systems, Man and Cybernetics, Vol. 15, No. 1, pp. 116-132.

Tsoukalas, L.H. and Uhrig, R.E. (1997). Fuzzy and Neural Approaches in Engineering, Wiley-Interscience.

Young, P.C. (1984). Recursive Estimation and Time-Series Analysis: An Introduction, Springer-Verlag. 